\title{
Preface
}

\section{Novel Approaches to the Management of Sleep- Disordered Breathing: Reviewing the Past and Looking Into the Future}

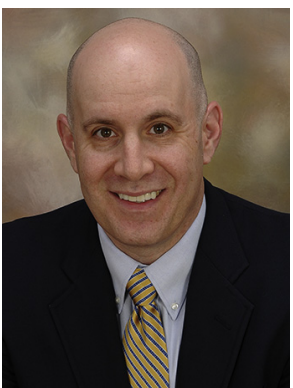

Neil Freedman, MD

Editor
Sleep-disordered breathing means different things to different people. While the term is often used interchangeably with obstructive sleep apnea (OSA), in a broader sense the term refers to the spectrum of breathing disorders that occur, or are exacerbated, during the sleep period. This issue of Sleep Medicine Clinics, written by some of the leaders in the field of sleep medicine, reviews many of the recent advancements in the management of sleep-disordered breathing as well as looks into the future of how these disorders will likely be managed.

The majority of the issue is dedicated to the management of OSA. OSA is common, and its prevalence is increasing as our population ages and becomes more overweight. The standard approach to the diagnosis and treatment of OSA involves one or two technician-attended sessions in the sleep lab, typically resulting in the initiation of continuous positive airway pressure (CPAP) therapy. This approach, while comprehensive, is costly and has consistently resulted in CPAP adherence rates of only $50 \%$ to $60 \%$. The relatively recent introduction of an out-of-center management approach of OSA with home sleep testing and AutoCPAP has been associated with similar outcomes to the standard approach. While these newer management approaches result in reduced costs, they have not been associated with improved adherence to therapy or improvements in other important outcomes. Thus, newer approaches to the diagnosis and treatment of OSA are needed.

In this issue, Drs Sands, Owens, and Malhotra initially review the physiology of OSA and examine several current and potential future diagnostic approaches to this disease. The following articles by Drs Kuzniar and Morgan are devoted to advances in the standard OSA treatments, such as CPAP and oral appliance therapies. Dr Hwang reviews how telemedicine and mobile applications may augment and potentially improve how clinicians manage and potentially improve important outcomes with positive airway pressure (PAP) therapy. Surgery can be considered for select groups of patients who are not candidates for, or who cannot tolerate, PAP or oral appliance therapies. In his article, Dr Soose reviews novel surgical approaches to the treatment of OSA. Dr White then reviews current and potential pharmacologic approaches to the treatment of OSA. Finally, two articles are dedicated to reviewing how precision medicine and big data may be used in the future to aid in the management of OSA.

Central sleep apnea (CSA) defines another spectrum of sleep-related breathing disorders characterized by cessations of airflow in the absence of respiratory effort. CSA can be 
observed in several medical disorders, and a specific type of CSA has been associated with increased morbidity and mortality in patients with congestive heart failure with reduced ejection fraction. Some initial post-hoc analysis data from a large randomized trial demonstrated that CPAP treatment of CSA, in a subgroup of CHF patients, resulted in improved survival and cardiac function. Unfortunately, results from a more recent large randomized trial of adaptive servo ventilation PAP therapy in this patient population demonstrated increased mortality and no improvements in hospitalizations, symptoms, or functional capacity. Thus, newer and better approaches are required to improve outcomes in these patients. Dr Javaheri's article reviews several novel therapies for the management of CSA.

Finally, sleep-related breathing disorders are not limited to OSA and CSA. Chronic obstructive pulmonary disease (COPD) can be associated with several types of sleep-related breathing disorders. Some data suggest that untreated patients with the overlap syndrome (COPD with OSA) have more exacerbations of their chronic lung disease, more hospitalizations, and an overall higher mortality compared with patients with COPD without concomitant OSA. These observational studies also suggest that CPAP treatment can improve many of these outcomes. In addition, there are conflicting data on whether more innovative PAP devices can reduce hospitalizations and improve survival in patients with more advanced lung disease. Drs Berrios and Gay review some of the novel management approaches to patients with COPD and associated sleep-disordered breathing.

As noted previously, we have made many advances in the management of the spectrum of sleep-related breathing disorders. Despite these advances, we still have a long way to go. We need better ways to identify and diagnose at-risk patients and determine the best treatment methods to improve important patient outcomes. This issue of Sleep Medicine Clinics should offer the reader some insight into the future of managing these patients and disorders. Enjoy!

Neil Freedman, MD Division of Pulmonary Critical Care, Allergy, and Immunology NorthShore University Health System 2650 Ridge Avenue Evanston, IL 60201, USA

E-mail address: nfreedman@northshore.org 